\title{
ACERCA DEL CAMBIO DE PARADIGMA Y LA EXISTENCIA DEL UNUNSEPTIUM
}

\author{
María de la Merced Velázquez Quintana \\ (UACH y UNAM, México)
}

\begin{abstract}
Resumen
El presente ensayo es una reflexión sobre el descubrimiento de un nuevo elemento químico y su impacto en el paradigma de una ciencia. Fue realizado en el contexto del artículo de Covarrubias (2010), quien aborda el tema de la construcción de los cuerpos teóricos, en referencia los trabajos de Kuhn.
\end{abstract}

Palabras clave: Filosofía de la ciencia | Conocimiento | Química

\begin{abstract}
This essay is a reflection on the discovery of a new chemical element and its impact on the paradigm of a science. It was made in the context of the article of Covarrubias (2010), who addresses the issue of the construction of the theoretical bodies, in reference works of Kuhn.
\end{abstract}

Keywords: Philosophy of science | Knowledge | Chemistry

\section{Introducción}

"Medir exactamente un objeto fugaz o indeterminado, medir exactamente un objeto fijo y bien determinado con un instrumento grosero, he ahí dos tipos de ocupación vana que la disciplina científica rechaza de inmediato" (Bachelard 2004:250, apud Covarrubias 2010)

La construcción de conocimiento científico se caracteriza por procesos de hegemonización de teorías. Una teoría se convierte en paradigma cuando la comunidad científica la acepta como un sistema repleto de enigmas, los cuáles se ocupa de resolver a través de la investigación. Los problemas que no pueden ser resueltos, constituyen las anomalías. 


\section{Desarrollo del tema}

El carácter existencial de cada teoría implica una lógica determinada, que no puede ser leída por cualquier epistemología, sino solamente por aquella que se desprende de su propia ontología. La definición de enigmas y anomalías en la teoría dominante, conduce a una crisis y al surgimiento de otras teorías que plantean posibles soluciones, hasta que, finalmente, una de ellas logra ocupar el sitio hegemónico.

Por otra parte, la teorización de una anomalía no está referida al grado de correspondencia que tenga con los objetos reales a los que alude, sino con la presencia de referentes, que implican su racionalidad en la conciencia de los científicos encargados de su teorización. De este modo, a mayor número de referentes, existe mayor posibilidad de que la teoría sea aceptada y de que convierta en paradigma.

También es posible una racionalidad diferente a la del paradigma, ya sea porque en la conciencia del científico existan referentes de teorías no vigentes, o porque se activen referentes ateóricos, los cuales tienen gran impacto en la percepción de formas y contenidos.

Además, en ocasiones, lo que es percibido en algún supuesto contenido de lo real, es llevado a una generalización tal, que logra convertirse en ley, olvidando que se trata de un constructo de la razón y no de un contenido real. La fenomenología considera que únicamente existen los contenidos de la conciencia, en una posición opuesta al realismo, que admite solamente los contenidos de lo real.

En todo proceso de construcción de conocimiento científico, se opera con la lógica de la conciencia del investigador, que no es otra que la lógica de las teorías dominantes en un momento histórico-social, de modo tal que lo percibido y el instrumental usado para la verificación de su objetividad pertenecen a la misma teoría.

La ciencia actual tiene una gran devoción por la precisión de la medida. Bachelard (apud Covarrubias) hace al respecto la siguiente afirmación: "En efecto, una de las exigencias primordiales del espíritu científico, es que la precisión de una medida debe referirse constantemente a la sensibilidad del método de medida y que ha de tener en cuenta naturalmente las condiciones de permanencia del objeto medido. Medir exactamente un objeto fugaz o indeterminado, medir exactamente un objeto fijo y bien determinado con un instrumento grosero, he ahí dos tipos de ocupación vana que la 
disciplina científica rechaza de inmediato" (Bachelard 2004:250).

Un claro ejemplo de enigma, anomalía y cambio de paradigma fue recientemente noticia mundial. El 1 de mayo de 2014, la revista Phisical Review Letters (Khuyagbaatar, 2014) confirmó la existencia de un nuevo elemento químico. Se trata de un elemento sintético superpesado, el cual fue reportado desde el año 2010, por el Joint Institute for Nuclear Research de Dubna, Rusia. (Oganessian, 2010)

Dicho elemento fue creado al colisionar isótopos de calcio-48 contra isótopos de berkerelio-249. Esta maniobra generó un nuevo elemento integrado por 117 protones. El experimento logró replicarse hasta cuatro años más tarde, en Alemania, cumpliendo entonces el requisito de la confirmación por un laboratorio independiente. De esa manera, se probó la existencia del nuevo elemento, que ahora ocupa el lugar 117 en la tabla periódica y al cual se asignó un nombre provisional en el idioma latín: ununseptium.

Debe mencionarse que entre los elementos químicos, la estabilidad de los núcleos disminuye en forma progresiva conforme aumenta su número atómico, a partir del plomo (número atómico 82). Además, los elementos con un número atómico mayor a 101, muestran decaimiento radioactivo y una vida media menor a un día. Únicamente los elementos con número atómico del 110 al 114 tienen mayor estabilidad nuclear, por lo cual, dicha zona se conoce como la "Isla de la estabilidad".

Aunque algunos elementos eran conocidos desde la antigüedad, el primero es ser descubierto utilizando el método científico fue el fósforo ("Portador de luz"), en el siglo XVII, por el alquimista Henning Brand. El siguiente siglo favoreció el descubrimiento de los gases, a los cuáles siguieron los metales alcalinos y los alcalino-terráqueos, facilitados por el invento de la pila eléctrica.

A mediados del siglo XIX, con el desarrollo del espectroscopio, se descubrieron otros elementos, los cuáles fueron nombrados según el color de sus espectros obtenidos en este equipo: cesio, en referencia al color azul; talio por el color verde, similar al de los tallos, y rubidio, por su espectro rojo.

La creación del ununseptium constituyó un enigma o punto de partida para hacer la investigación que condujo a su confirmación. Su existencia como el segundo elemento más pesado que existe, el cual es radioactivo y tiene una vida de apenas 50 milisegundos, puede ser considerada una nueva anomalía en la teoría de los elementos químicos.

En tono a esta teoría química, se aprecia un cambio en diferentes paradigmas: ya no 
se trata descubrir un elemento que existe en la naturaleza, sino de su creación. También su corto tiempo de vida seguramente obligará a modificar los instrumentos necesarios para su confirmación y manejo.

\section{Bibliografía}

Covarrubias, F. (2010). El proceso de construcción de corpus teóricos: la importancia de los referentes no teóricos en los procesos de teorización. Cinta Moebio, 37 1, 15-28.

Khuyagbaatar, J. (2014). 48Ca $+249 \mathrm{Bk}$ fision reaction leading to element $\mathrm{Z}=117$ : longlived a decaying 270 Db and Discovery of 266Lr. Phys Rev Lett, 172501.

Oganessian, Y. (2010). Synthesis of a new element with atomic number $\mathrm{Z}=117$. Phis Rev Lett, 142502. 